\title{
Efecto de la Representación Dinámica Integrada sobre la competencia matemática y la atención en niños con Trastorno por Déficit de Atención e Hiperactividad
}

\author{
Nuria Peláez', Marisol Cueli', Débora Areces', Trinidad García’ y Celestino Rodríguez * \\ 'Universidad de Oviedo
}

\begin{abstract}
Resumen. El Trastorno por Déficit de Atención con o sin Hiperactividad (TDAH), ha sido objeto de numerosos estudios. Uno de los aspectos más destacados del trastorno, es su posible comorbilidad con las dificultades de aprendizaje de las matemáticas lo que pone en relevancia la necesidad de intervenciones dirigidas a trabajar estos aspectos. El objetivo de este estudio fue analizar la eficacia de una intervención, la Representación Dinámica Integrada, en la mejora de variables relacionadas con las competencias matemáticas (evaluadas con el TEMA-3 y la resolución de problemas) y atencionales (evaluadas con las pruebas RAN/RAS y CARAS-R) en estudiantes con TDAH. Participaron 23 estudiantes de entre 6 y 9 años. Los resultados mostraron que la herramienta resultaba eficaz en la mejora de las variables analizadas, sobre todo, las competencias informales y la velocidad de nombramiento de objetos números y letras. En conclusión, la herramienta resultó eficaz en la mejora de la competencia y en las variables atencionales, aspectos clave en los estudiantes con TDAH y dificultades en matemáticas.
\end{abstract}

\section{Palabras clave: TDAH, atención, competencia matemática, Representación Dinámica Integrada.}

Effect of Integrated Dynamic Representation on Mathematical Competence and Care in Children with Attention Deficit with Hyperactivity Disorder.

Abstract. While numerous studies have addressed the comorbidity of ADHD with reading and writing difficulties, very few have focused on the relationship between ADHD and difficulties in mathematics, with a high rate of comorbidity. This study aims to determine the effectiveness of a new tool (Integrated Dynamic Representation-IDR, or RDI in Spanish) to improve basic mathematics skills (assessed by means of the TEMA-3 scale) and attention (naming speed variables, evaluated through the administration of rapid RAN / RAS; and selective attention, by using CARAS). For this purpose, an experimental group of 23 students (age range $=6-9$ years) took part in this study. Results showed that mathematics informal skills and naming speed were the most improved components after the intervention, mainly regarding comparison and naming of objects, numbers and letters. Therefore, the tool that was tested (RDI), showed to be effective to improve mathematical skills, as well as attention, which are core aspects in the case of students with ADHD and mathematics difficulties.

Keywords: ADHD, attention, mathematical competence, Integrated Dynamic Representation.

Actualmente, la nueva versión del Manual Diagnóstico y Estadístico de los Trastornos Mentales -DSM 5- (American Psychiatric Association, 2013), describe el Trastorno por Déficit de Atención con o sin Hiperactividad

Recibido: 30/04/2017 - Aceptado: 25/05/2017 - Avance online: 20/06/2017 *Correspondencia: Celestino Rodríguez.

Universidad de Oviedo, Plaza Feijoo s/n.

C.P: 33003, Oviedo, España.

E-mail: rodriguezcelestino@uniovi.es
(TDAH) como un trastorno neurobiológico que afecta tanto a adultos como a niños y que se caracteriza por un patrón persistente de inatención y/o hiperactividad e impulsividad. Cabe destacar, que esta nueva versión del DSM, incluye algunas modificaciones relativas a la edad de aparición, a la terminología empleada en referencia al trastorno y a las posibles comorbilidades con las que se 
presenta. En primer lugar, si bien en versiones anteriores del manual (DSM-IV-TR; American Psychiatric Association, 2000) la edad de aparición de la sintomatología se situaba en el límite de los 7 años, actualmente el criterio se ha incrementado hasta los 12 años de edad. En segundo lugar, en referencia a la terminología empleada, el concepto "subtipo" ha sido sustituido por el de "tipo de presentación". De esta forma, en el DSM 5 se establece que el TDAH puede manifestarse en función de tres tipos de presentación que pueden variar a lo largo de la vida del individuo, presentación combinada, presentación predominantemente inatenta y presentación predominantemente hiperactiva/impulsiva. En tercer lugar, con respecto a la comorbilidad, el TDAH ha sido incluido ahora dentro de los trastornos del neurodesarrollo, en lugar de los trastornos conductuales, destacándose su posible comorbilidad con otras sintomatologías como el Trastorno del Espectro Autista o las Dificultades de Aprendizaje.

En esta línea, autores como Mayes, Calhoum y Crowell (2000) han destacado que el TDAH y las DA se presentan de forma combinada en un porcentaje que varía entre el 25 y el $35 \%$ de los casos. Concretamente, en el área de las matemáticas, sitúan este porcentaje en el $26 \%$ de los casos (Mayes et al., 2000). Sin embargo, autores como Miranda, Meliá y Marco (2009), apuntan que el número de estudios centrados en la comorbilidad entre el TDAH y las dificultades de aprendizaje en matemáticas (DAM) es inferior a las investigaciones realizadas en dificultades de aprendizaje en lectoescritura. También, Lucangeli y Cabrele (2006) resaltan que las investigaciones previas se han centrado principalmente en la comorbilidad con las dificultades en la lectura en comparación con las DAM y, sobre todo, con las dificultades en el área de resolución de problemas.

En relación con las DAM, diferentes autores (Gaitán-Chipatecua y Rey-Anacona, 2013; Sattler y Hoge, 2008) han afirmado que estas dificultades se presentan ya en las habilidades matemáticas básicas (por ejemplo el conteo, la comparación de cantidades, el aprendizaje de las tablas de multiplicar,...), en la atención (por ejemplo en el signo de la operación o en la recogida correcta de los datos), y en el procesamiento perceptual o espacial (de la relación entre los datos numéricos relevantes). En consecuencia, este grupo de estudiantes tiene serias dificultades para resolver problemas matemáticos, por lo que precisan del uso de estrategias que faciliten la comprensión del enunciado de partida y su resolución posterior. Por su parte, Kaufman y Nuerk (2008) indicaron que los niños con TDAH presentan un nivel de desarrollo significativamente inferior al de sus compañeros en las habilidades básicas de procesamiento numérico $y$, si bien en la población general, el bajo rendimiento en matemáticas decrece con la edad, en el alumnado con TDAH tiende a aumentar (Kroesbergen, Van Luit, y Aunio, 2012). Además, estas dificultades se incrementan cuando ambas sintomatologías (TDAH y DAM) se presentan de forma conjunta. En este sentido, investigaciones como la de Meliá (2008), coinciden en afirmar que niños con TDAH y DAM muestran un rendimiento significativamente inferior al de aquellos estudiantes sin dificultades en tareas sencillas de cálculo, de recuento de puntos, de comparación de polígonos y de series numéricas.

En cuanto a la resolución de problemas, Krawec (2010) observó que, los estudiantes con DAM tienen dificultades para identificar la información relevante presente en el enunciado de un problema y que permite alcanzar la resolución final. Concluyó a su vez que, el alumnado con DAM muestra un déficit en la discriminación, que afecta a su capacidad para resolver los problemas adecuadamente. Dadas estas dificultades, se pone en relevancia la necesidad de llevar a cabo intervenciones ajustadas y eficaces. Para ello, las investigaciones apuntan a la importancia del uso de las nuevas tecnologías en este ámbito (Engel y Onrubia, 2013; Lazakidou y Retalis, 2010).

En el ámbito tecnológico, destacan principalmente dos herramientas. Por una parte, se encuentra la empresa Rehasoft, que en colaboración con el Instituto de Pedagogía y Educación Especial de Suecia, ha creado el programa MeMotiva en el año 2005 (De Marco, 2010) para aumentar la capacidad 
de la memoria operativa tanto en niños como en adultos con dificultades de atención y de concentración. Este programa, ha mostrado su eficacia en investigaciones previas como la realizada Semrud-Clikeman y Bledsoe (2011) quienes concluyeron una mejora significativa en los estudiantes con TDAH en comparación con aquellos que no había realizado la intervención con el citado programa. Por otra parte, González-Castro, Cueli, Cabeza, Álvarez-García y Rodríguez (2014) han utilizado la herramienta Representación Dinámica Integrada (RDI) para intervenir sobre las habilidades matemáticas básicas informales y formales. Los resultados de su investigación, mostraron una mejora significativa tras la intervención con la RDI en estudiantes sin dificultades de aprendizaje. En todo caso, en el citado trabajo, se expone la necesidad de concretar los beneficios de esta herramienta en alumnado con DAM y/o con TDAH.

La Representación Dinámica Integrada (RDI; González-Castro, Cueli et al., 2014) está dirigida a entrenar las competencias matemáticas básicas (informales y formales) y la resolución de problemas de manera conjunta en edades tempranas. La herramienta se estructura en torno a tres componentes: Comprensión fragmentada, representación fragmentada e integración del conjunto de representaciones siguiendo el modelo de Mayer (2004) (traducción, integración, planificación y ejecución), en función del cual, se establece un proceso de aplicación de cuatro niveles de representación: Representación de los conceptos, representación de las relaciones, representación de los interrogantes, reversibilidad del proceso (generalización a otros contextos). La RDI sigue la secuencia lógica a la hora de aplicar las competencias propias del nivel educativo en el que se enmarca el programa. Los contenidos se presentan siguiendo tres tipos de presentación de la información, la presentación icónica (los conceptos se presentan asociados a imágenes), presentación combinada (los conceptos van asociados a imágenes/palabras) y la presentación simbólica (los enunciados se presentan exclusivamente en texto lineal). Así, se trabajan competencias previas al currículo como son las competencias informales: Numeración (el conteo, teniendo en cuenta que el número crece y decrece a medida que aumenta o disminuye el número de objetos), la comparación de cantidades (dado que en la representación del enunciado los datos numéricos quedan reflejados en el número concreto de objetos en cada uno de los bolos), cálculo informal (la resolución del problema sin realizar la operación concreta sino arrastrando los objetos al último de los bolos de solución final), concepto informal (el todo incluye a las partes y el niño arrastra el número de objetos representado en el dato numérico). También competencias propias del currículo como son las competencias formales: Convencionalismos (codificación y decodificación del número; el número aparece de forma simbólica y escrita), hechos numéricos (cálculo mental; en este caso, aún no se introduce la operación sino que la solución aparece al colocar la operación en el lugar correspondiente), cálculo formal (realización de la operación mecánica), y concepto formal (concepto simbólico de número; el todo incluye a las partes y el niño ya no arrastra el número de objetos representado en el dato numérico sino un solo objeto). Todo ello, se estructura de forma secuencial y jerarquizada en 9 niveles de dificultad creciente. Estos niveles, incluyen inicialmente problemas de sumas sin llevadas, a continuación con llevadas y restas sin llevadas, para progresivamente introducir la combinación de sumas y restas. Al mismo tiempo se va incorporando el concepto de número en intervalos que van de $1-3,0-5,0-9$, 0-19, 0-39, etc. En todos los niveles se realiza la reversibilidad del proceso, de esta forma se fomenta que lo aprendido se generalice a otros contextos. La reversibilidad consiste en que el sujeto debe formular el problema teniendo presente lo que él ha hecho previamente.

El objetivo principal de este trabajo es determinar la eficacia de la RDI en el alumnado con TDAH. Para ello, se han concretado dos objetivos específicos. El primero de ellos es analizar la mejora en la competencia matemática de los niños que reciben la intervención; y el segundo es analizar si tal mejoría se generaliza a otros aspectos como las habilidades atencionales. 
Teniendo en cuenta estos objetivos, se han planteado tres hipótesis. La primera hipótesis, referida al primer objetivo, es que la herramienta RDI resultará eficaz en la intervención en el alumnado con TDAH. Centrándose en los objetivos específicos, la segunda hipótesis plantea que se producirá una mejora en la competencia matemática tras la intervención con la RDI en el alumnado con TDAH. Por último, la tercera hipótesis afirma que se producirán cambios en las medidas atencionales tras la intervención realizada.

\section{MÉTODO}

\section{PARTICIPANTES}

En este estudio se dispuso de una muestra de 23 estudiantes, 15 alumnos (65.2\%) y 8 alumnas (34.8\%), de entre 6 y 9 años de edad $(M=7.2$ años; $D T=1.16)$ y con un $\mathrm{Cl}$ igual o superior a $85(M=102.83 ; D T=13.09)$. Los niños presentaban una edad media de 7.27 años $(D T=1.16)$ y un $\mathrm{Cl}$ medio de 105.73 (DT $=14.19$ ). En cuanto a las niñas, la edad media era de 8.00 años $(D T=1.07)$ y el $\mathrm{Cl}$ medio de $97.38(D T=9.14)$. El total de los 23 estudiantes formaba parte del grupo experimental.

Todos los participantes estaban diagnosticados con TDAH (en cualquiera de sus presentaciones) con un informe clínico. Dicho diagnóstico fue contrastado mediante las pruebas BRIEF (Gioia, Isquith, Guy, y Kenworthy, 2000) y SNAP-IV (DuPaul, Anastopoulos, Power, Reid, Ikeda, y McGoey, 1998), clasificando a 17 estudiantes (73.9\%) en la presentación combinada y a 6 estudiantes $(26.1 \%)$ en la predominantemente inatenta. Ninguno de los estudiantes estaba diagnosticado con la presentación predominantemente hiperactiva/ impulsiva. Del total de la muestra, 18 de los participantes (78.3\%) recibían medicación que no fue suspendida durante la evaluación ni la intervención.

El método de selección de la muestra fue no probabilístico, siendo elegidos los participantes en función de la disponibilidad y accesibilidad. Todos formaban parte de la Asociación de Niños Hiperactivos del Principado de Asturias (A.N.HI.P.A).

\section{INSTRUMENTOS DE MEDIDA}

Para la descripción de la muestra se han utilizado tres pruebas, el FACTOR G, el SNAP IV y el BRIEF.

El FACTOR G (Cattell y Cattell, 1995) es un test para medir la capacidad intelectual sin la influencia de elementos culturales. La escala 1 fue aplicada a los niños hasta los 8 años y la escala 2 a los niños a partir de esa edad. La escala 7 consta de 8 subpruebas, de las cuales se realizaron 4 (sustitución, clasificación, laberintos y semejanzas), que son las recomendadas para evitar la influencia cultural. La escala 2 consta de 4 subpruebas, todas ellas libres de influencia cultural, series, clasificación, matrices y condiciones.

El SNAP IV (DuPaul et al., 1998) es una escala dirigida a padres y profesores (en el presente estudio se ha utilizado la versión para padres) que consta de 18 ítems (en su versión abreviada) y aporta información sobre la presencia o ausencia de los síntomas del TDAH siguiendo los criterios del DSM-IV-TR (APA, 2000). El coeficiente de fiabilidad para la escala de los padres es de 0.88 (Servera y Cardo, 2007).

El BRIEF (Gioia et al., 2000) es un cuestionario dirigido tanto a padres como a profesores. En el presente estudio se ha utilizado la versión para padres. El cuestionario consta de 27 ítems y aporta información sobre las funciones ejecutivas, como son la impulsividad, hiperactividad, control emocional, memoria funcional, planificación, organización, cambio, concentración y focalización. Este instrumento tiene una alta consistencia interna, en torno a 0.90 (García et al., 2013).

Con el fin de analizar la eficacia de la intervención se realizaron pruebas a nivel atencional (incluyendo la percepción de diferencias y la velocidad de nombramiento) y matemático (evaluando tanto la competencia matemática como la resolución de problemas en su resultado y proceso).

El Test de Percepción de Diferencias-Revisado CARAS-R (Thurstone y Yela, 2012), tiene como objetivo evaluar los aspectos perceptivos y atencionales de estudiantes a partir de los seis años de edad. La prueba cuenta con 60 
elementos, cada uno formado por tres dibujos que representan caras con trazos básicos (pelo, cejas, ojos y boca). Dos de estas caras son iguales y la tercera tiene una diferencia. El estudiante debe analizar el elemento y señalar cuál es la cara diferente. La duración de la prueba es de tres minutos. El CARAS-R además de recoger los aciertos y errores, proporciona dos variables, los aciertos netos A-E (resultado de la resta aciertos - errores), y el índice de control de la impulsividad (ICI) gracias al cual podremos relacionar el rendimiento con el estilo de respuesta (Crespo-Eguílaz, Narbona, Peralta, y Repáraz, 2014). La prueba presenta un índice de fiabilidad de 0.91.

El RAN/RAS (Wolf y Denckla, 2005) evalúa la velocidad de nombramiento en seis láminas o tareas específicas (nombramiento de objetos, colores, letras, números, combinación de letras-números, y combinación de letrasnúmeros-colores). La variable registrada es el tiempo (en segundos) que el estudiante requiere para el nombramiento de cada una de las seis láminas. Las variables incluidas en el estudio fueron los tiempos pen la denominación de las seis láminas o tareas específicas.

El Test de Competencia Matemática Básica (Ginsburg y Baroody, 2003). Es una prueba que evalúa las competencias matemáticas informales (aquellas aprendidas fuera del contexto escolar) y formales (aquellas aprendidas en el contexto escolar reglado). Las informales se miden mediante numeración (representación de cantidades y cálculo mental), comparación (manejo del crecimiento/ disminución de los números), cálculo informal (resolución de operaciones de suma y resta) y concepto informal (agrupar conjuntos mediante manipulación). Las competencias formales se evalúan a través de convencionalismos (leer y escribir cantidades), hechos numéricos (operar mediante suma, resta y multiplicación), cálculo formal (sumas y restas de dificultad creciente) y concepto formal (identificar el significado numérico desde el punto de vista simbólico e icónico). Este instrumento ofrece el Índice de Competencia Matemática (ICM) que indica el rendimiento global del alumno en relación al grupo de edad al que pertenece. Las variables incluidas en el estudio fueron las referidas a las cuatro competencias informales y la puntuación global de pensamiento informal, las cuatro competencias formales y la puntuación global de pensamiento formal y el ICM. El alfa de Cronbach del total de la prueba es de 0.92.

Los problemas de razonamiento utilizados se han basado en la estructura seguida por Riley, Greeno y Heller (1983). La evaluación se ha basado tanto en el resultado (registro de aciertos y errores) como en el proceso (caracterizado por la "planificación" o "ejecución"). Para ello, se han utilizado cuatro problemas matemáticos, los dos primeros se resolvían por adición y los dos últimos por sustracción. Las variables incluidas en el estudio fueron, los aciertos y errores para el resultado y la "planificación" y "ejecución" para el proceso. Para el análisis del proceso se tuvo en cuenta el modelo de autorregulación de Zimmerman (2000, 2008), en función del cual, los estudiantes autorregulados dirigen su aprendizaje a través de la puesta en práctica de una serie de estrategias, activando y modificando sus procesos cognitivos, metacognitivos y comportamentales, antes, durante y después de que el aprendizaje tenga lugar y siguiendo las fases de planificación, ejecución y evaluación del proceso. Teniendo esto en cuenta, se determinó como "planificación" cuando el estudiante identificaba el problema, anotaba los datos, elegía una estrategia de resolución y la llevaba a cabo. Se clasificó como "ejecución" cuando el sujeto exploraba las diferentes estrategias y elegía una para realizar el problema.

\section{PROGRAMA DE INTERVENCIÓN: REPRESENTACIÓN DINÁMICA INTEGRADA (RDI)}

Como herramienta de intervención se utilizó la RDI desarrollada por Álvarez, GonzálezCastro, Núñez y González-Pienda (2007). Esta estrategia es el elemento nuclear de los procesos heurísticos y resulta de la combinación de representaciones externas e internas. Se estructura en torno a tres componentes (SolazPortolés y Sanjosé-López, 2008): comprensión fragmentada, representación fragmentada e integración de las representaciones. 
El proceso de aplicación se concreta en la RDI en cuatro niveles de representación. El nivel número 1 es la Representación de los conceptos (Selección de la información relevante) en el que se presenta el enunciado del problema destacando los conceptos clave, asociados a dibujos, los datos numéricos que los acompañan se enmarcan en cuadrados y los verbos se sustituyen por pictogramas. El nivel número 2 es la Representación de los enlaces (fragmentación), en el que una vez identificados los conceptos clave, estos se representan en conjuntos unión-intersección, cuyo número de elementos va concretando los datos numéricos. En el nivel número 3, la Representación de los interrogantes (Integración de las representaciones). En este tercer nivel las representaciones se conectan entre sí, según sean los tipos de relación que marquen los enlaces del enunciado: unión (suma) e intersección (resta). Por último, en el nivel número 4, la Reversibilidad del proceso (Generalización a otros contextos), en base al cual, a partir de la representación integrada que lleva a la solución final, y sin tener presente el enunciado de partida, se le pide al sujeto que reformule de nuevo el enunciado del problema. Esta estrategia favorece la reversibilidad y, por lo tanto, la generalización de lo aprendido.

El manejo del programa a nivel informático resulta sencillo y está adaptado a las habilidades del alumnado por edad y nivel educativo. Todo ello se plantea siguiendo tres tipos de representación: icónica (los conceptos van asociados a imágenes), combinada (los conceptos van asociados a imágenes/palabras) y simbólica (los enunciados se presentan exclusivamente en texto lineal).

\section{PROCEDIMIENTO}

En primer lugar, una vez planteados los objetivos y establecido las pruebas necesarias, nos ponemos en contacto con la Asociación de Niños Hiperactivos del Principado de Asturias (A.N.HI.P.A) con el fin de informales de los objetivos de la investigación y solicitar su colaboración y las de las familias para lo cual se envió una carta informativa. Una vez obtenido el consentimiento activo de la asociación y las familias, se procedió a recoger la información pretest. El pretest se realizó en dos sesiones de aproximadamente 50 minutos para cada uno de los participantes. Estas sesiones se llevaron a cabo a lo largo de las tres primeras semanas de marzo del 2015 en la Facultad de Psicología de la Universidad de Oviedo. En la primera sesión se pasaban las pruebas FACTOR-G, el CARAS-R, y RAN/ RAS. En la segunda sesión se realizaba el TEMA-3 y los problemas de razonamiento. Durante las sesiones de evaluación, los padres cumplimentaban el BRIEF y el SNAP IV.

Una vez finalizada la evaluación pretest, se procedió a llevar a cabo la intervención durante los meses de abril y mayo, concretamente del 13 de abril al 22 de mayo del año 2015. La intervención se realizó en un total de 2 sesiones semanales de media hora cada una. Para realizar la intervención se elaboró un protocolo donde figuraban los materiales utilizados, la distribución de los grupos, así como los objetivos de cada sesión y el tiempo requerido. El mismo profesional guiaba y realizaba el seguimiento del proceso de ejecución de los estudiantes. Todos ellos iniciaban la intervención con la presentación icónica y progresivamente se iba incrementando el grado de dificultad. Finalmente, se realizó la evaluación postest en la última semana de mayo y la primera de junio del año 2015. Una vez realizadas todas las pruebas, se procedió a la corrección de las mismas y al volcado de datos con el fin de proceder a su análisis estadístico.

\section{DISEÑO Y ANÁLISIS DE DATOS}

Se utilizó un diseño pre-experimental, compuesto por un solo grupo (grupo experimental). Para alcanzar los objetivos propuestos, los datos fueron analizados en diferentes pasos. En primer lugar se analizaron los estadísticos descriptivos (medias, desviaciones típicas y medidas de asimetría y curtosis para las variables de competencia matemática y atencionales). Teniendo en cuenta el criterio de Finney y Di Stefano (2006), según el cual se considera que las variables cumplen los criterios de normalidad cuando los valores para la asimetría y la curtosis se 
encuentran entre \pm 2 y \pm 7 , se puede concluir que todas las variables cumplían estos criterios, excepto las relacionadas con la velocidad de nombramiento.

Dado el cumplimiento de los criterios de normalidad, se realizó la prueba $t$ de Student para las variables de competencia matemática del TEMA-3 y para las variables del test de percepción de diferencias CARAS-R. Para el análisis de la velocidad de nombramiento obtenida del test RAN/ RAS se realizó la prueba no paramétrica de Wilcoxon. Para el análisis de la resolución de problemas, se registró tanto el resultado como el proceso de resolución. Para el análisis del resultado (acierto / error) se llevó a cabo la prueba de McNemar al tratarse de una variable dicotómica, teniendo en cuenta para el análisis del proceso (planificación / ejecución), el estadístico chi cuadrado $\left(x^{2}\right)$.

Para calcular el tamaño de las diferencias y valorarlas, se ha tenido en cuenta el criterio establecido en el trabajo clásico de Cohen (1988), el cual recoge una $d \geq .20$ es indicativa de un tamaño de las diferencias pequeño, $d \geq .50$ refleja un tamaño medio y se considera grande si $d \geq .80$. Finalmente, para la realización de los análisis estadísticos, se ha utilizado el paquete estadístico SPSS en su versión 22.0 (Arbuckle, 2010).

\section{RESULTADOS}

\section{RESULTADOS EN LA COMPETENCIA MATEMÁTICA}

A continuación se presentan los resultados en relación a la competencia matemática, analizados utilizando el estadístico $t$ de Student para las variables del TEMA-3, y el estadístico McNemar para las variables referidas al resultado en los problemas matemáticos. Como se puede ver en la tabla 1, los resultados aportados por la prueba $t$ de Student mostraron diferencias estadísticamente significativas entre el pretest y el postest en las variables numeración, comparación, concepto informal, pensamiento informal, convencionalismos, hechos numéricos y pensamiento formal, con un tamaño del efecto comprendido entre 0.30 (pequeño) y 0.78 (medio), correspondiente a la variable comparación.

Además, delanálisis del proceso de resolución de los cuatro problemas matemáticos, se puede ver que, tras la intervención se han producido cambios en la manera en la que los estudiantes realizan los problemas; concretamente en la realización de los problemas 1, 3 y 4 . En referencia a los aciertos y los fallos en cada problema, no se han detectado producido diferencias estadísticamente significativas tal y como se muestra en la Tabla 2.

Tabla 1

Medias, desviaciones típicas, asimetría, curtosis, prueba † de Student y d de Cohen para las variables del TEMA3, antes y después de la intervención

\begin{tabular}{|c|c|c|c|c|c|c|c|c|c|c|c|}
\hline & \multicolumn{4}{|c|}{ Pretest } & \multicolumn{4}{|c|}{ Postest } & \multirow[b]{2}{*}{$\boldsymbol{t}$} & \multirow[b]{2}{*}{$\boldsymbol{p}$} & \multirow[b]{2}{*}{$d$} \\
\hline & $M$ & DT & Asim & Curt & $M$ & DT & Asim & Curt & & & \\
\hline ICM & 80.13 & 17.91 & -0.10 & -0.97 & 80.35 & 26.61 & -0.88 & 2.476 & -0.07 & .948 & .01 \\
\hline \multicolumn{12}{|l|}{ Informales } \\
\hline Numeración & 19.61 & 2.57 & -1.40 & 2.02 & 20.83 & 2.04 & -1.79 & 2.90 & -3.102 & .005 & .54 \\
\hline Comparación & 4.70 & 0.70 & -0.35 & 0.41 & 5.22 & 0.67 & -0.28 & -0.63 & -4.899 & $<.001$ & .78 \\
\hline Cálculo Informal & 5.04 & 0.93 & 1.03 & 4.58 & 5.13 & 0.82 & 0.85 & 0.97 & -0.699 & .492 & .10 \\
\hline Concepto Informal & 2.57 & 0.84 & 1.02 & -0.76 & 2.96 & 0.83 & 0.09 & -1.52 & -2.398 & .025 & .48 \\
\hline Pensamiento Informal & 31.91 & 4.40 & -0.68 & 1.15 & 34.13 & 3.68 & -0.56 & -0.21 & -3.872 & .001 & .56 \\
\hline \multicolumn{12}{|l|}{ Formales } \\
\hline Convencionalismos & 6.26 & 1.63 & -1.22 & 1.14 & 6.74 & 1.42 & -1.78 & 4.52 & -2.554 & .018 & .32 \\
\hline Hechos Numéricos & 1.96 & 2.06 & 1.47 & 2.34 & 2.83 & 2.73 & 1.03 & 0.20 & -2.127 & .045 & .37 \\
\hline Cálculo Formal & 2.52 & 2.11 & 1.02 & 1.26 & 2.96 & 2.69 & 1.20 & 0.70 & -1.189 & .247 & .19 \\
\hline Concepto Formal & 1.61 & 0.99 & 2.15 & 5.43 & 1.78 & 1.31 & 1.63 & 1.54 & -0.778 & .445 & .15 \\
\hline Pensamiento Formal & 12.35 & 5.97 & 0.75 & 0.50 & 14.30 & 7.41 & 0.95 & 0.37 & -2.140 & .044 & .30 \\
\hline
\end{tabular}




\begin{tabular}{|c|c|c|c|c|c|}
\hline \multicolumn{6}{|c|}{$\begin{array}{l}\text { Tabla } 2 . \\
\text { Frecuencias, forma de realización de los problemas de razonamiento y estadístico McNemar } x^{2} \text { antes y después de la intervención }\end{array}$} \\
\hline & & \multirow{2}{*}{ Pretest } & \multirow{2}{*}{ Postest } & \multicolumn{2}{|l|}{ McNemar } \\
\hline & & & & $x^{2}$ & $p$ \\
\hline \multirow[t]{2}{*}{ Problema 1} & Acierto & 18 & 19 & 2.273 & 1.000 \\
\hline & Fallo & 5 & 4 & & \\
\hline \multirow[t]{2}{*}{ Realización } & Planificación & 12 & 19 & 1.433 & .039 \\
\hline & Ejecución & 11 & 4 & & \\
\hline \multirow[t]{2}{*}{ Problema 2} & Acierto & 15 & 19 & 0.494 & .289 \\
\hline & Fallo & 8 & 4 & & \\
\hline \multirow[t]{2}{*}{ Realización } & Planificación & 14 & 18 & 4.480 & .219 \\
\hline & Ejecución & 9 & 5 & & \\
\hline \multirow[t]{2}{*}{ Problema 3} & Acierto & 7 & 8 & 5.957 & 1.000 \\
\hline & Fallo & 16 & 15 & & \\
\hline \multirow[t]{2}{*}{ Realización } & Planificación & 12 & 19 & 1.433 & .039 \\
\hline & Ejecución & 11 & 4 & & \\
\hline \multirow[t]{2}{*}{ Problema 4} & Acierto & 10 & 8 & 9.673 & .625 \\
\hline & Fallo & 13 & 15 & & \\
\hline \multirow[t]{2}{*}{ Realización } & Planificación & 9 & 18 & 4.107 & .004 \\
\hline & Ejecución & 14 & 5 & & \\
\hline
\end{tabular}

Tabla 3.

Medias, desviaciones típicas, asimetría, curtosis y Wilcoxon para las variables del RAN/RAS antes y después de la intervención.

\begin{tabular}{|c|c|c|c|c|c|c|c|c|c|c|}
\hline & \multicolumn{4}{|c|}{ Pretest } & \multicolumn{4}{|c|}{ Postest } & \multirow[b]{2}{*}{$\boldsymbol{Z}$} & \multirow[b]{2}{*}{$\boldsymbol{p}$} \\
\hline & $M$ & $D T$ & Asim & Curt & $M$ & $D T$ & Asim & Curt & & \\
\hline Objetos & 66.43 & 18.94 & 0.79 & -0.33 & 60.87 & 20.82 & 1.57 & 2.08 & -3.568 & $<.001$ \\
\hline Colores & 72.96 & 19.94 & 0.44 & 0.66 & 68.87 & 22.03 & 1.34 & 2.63 & -2.798 & .005 \\
\hline Números & 44.61 & 16.70 & 1.43 & 2.77 & 37.87 & 13.74 & 1.56 & 3.19 & -3.594 & $<.001$ \\
\hline Letras & 43.22 & 12.40 & 0.81 & 1.33 & 37.78 & 11.33 & 0.65 & 0.15 & -3.601 & $<.001$ \\
\hline Letras Números & 50.87 & 22.66 & 2.90 & 11.12 & 46.43 & 18.64 & 2.26 & 7.35 & -3.066 & .002 \\
\hline LNC & 55.83 & 21.68 & 2.61 & 9.18 & 52.96 & 25.51 & 2.80 & 10.36 & -2.376 & .017 \\
\hline
\end{tabular}

Tabla 4 .

Medias, desviaciones típicas, asimetría, curtosis, t de Student y $d$ de Cohen para las variables del CARAS-R antes y después de la intervención.

\begin{tabular}{|c|c|c|c|c|c|c|c|c|c|c|c|}
\hline & \multicolumn{4}{|c|}{ Pretest } & \multicolumn{4}{|c|}{ Postest } & \multirow[b]{2}{*}{$t$} & \multirow[b]{2}{*}{$p$} & \multirow[b]{2}{*}{$d$} \\
\hline & $M$ & $D T$ & Asim & Curt & $M$ & $D T$ & Asim & Curt & & & \\
\hline Aciertos & 19.83 & 6.17 & -0.47 & 0.59 & 24.30 & 6.94 & 0.46 & -0.58 & -3.995 & .001 & .70 \\
\hline Errores & 4.17 & 5.81 & 2.12 & 4.36 & 2.91 & 2.80 & 1.33 & 1.89 & 1.179 & .251 & .28 \\
\hline$A-E$ & 15.65 & 8.80 & -1.23 & 2.16 & 21.39 & 7.54 & 0.55 & -0.42 & -4.540 & $<.001$ & .72 \\
\hline $\mathrm{ICl}$ & 69.19 & 37.03 & -1.98 & 4.05 & 79.02 & 18.09 & 0.62 & -0.44 & -1.569 & 131 & .34 \\
\hline \multicolumn{12}{|c|}{$\begin{array}{l}\text { Nota: } M=\text { Media, } D T=\text { Desviación Típica, Asim }=\text { Asimetría, Curt }=\text { Curtosis, } t=t \text { de Student, } d=d \text { de Cohen; A-E }=\text { Aciertos net } \\
\mathrm{ICl}=\text { Índice de control de la impulsividad. }\end{array}$} \\
\hline
\end{tabular}




\section{RESULTADOS DE ATENCIÓN}

En la Tabla 3 se presentan los resultados obtenidos en relación a las seis variables de velocidad de nombramiento. Los resultados obtenidos mediante la $Z$ de Wilcoxon mostraron diferencias estadísticamente significativas entre el pretest y el postest en todas las variables del RAN/RAS.

En la Tabla 4 aparecen los resultados obtenidos tras la realización de la prueba $t$ de Student para muestras dependientes en las variables del CARAS-R, así como el tamaño del efecto de las mismas. Tanto los aciertos como los aciertos netos presentan una mejora significativa con un tamaño del efecto medio (0.70 y 0.72 respectivamente). En cuanto a los errores y al índice de control de la impulsividad ( $\mathrm{Cl}$ ) no se observaron diferencias estadísticamente significativas.

\section{DISCUSIÓN}

Con la realización de este estudio se pretendió analizar los beneficios y la eficacia de la herramienta matemática RDI, en niños con TDAH. Concretamente, se pretendía determinar la eficacia de la RDI en el área de las matemáticas (mediante tareas de competencia matemática y resolución de problemas) y en el área de la atención (mediante tareas de velocidad de nombramiento y de percepción de diferencias). En concreto, se plantearon dos objetivos específicos que se discuten a continuación.

En cuanto al primer objetivo específico, en la muestra y en el contexto utilizado, la RDI mostró buenos resultados. Las diferencias observadas fueron estadísticamente significativas entre el pretest y el postest en la mayoría de las variables analizadas. Globalmente, el cambio más pronunciado se produjo en las competencias informales y en la velocidad de nombramiento. Estos resultados son compatibles con los obtenidos por González-Castro, Cueli et al. (2014), quienes detectaron que las competencias informales presentaban un cambio significativo mayor en los estudiantes sin dificultades de aprendizaje. Cabe destacar en relación a la resolución de problemas, el cambio detectado en el proceso de resolución por encima del resultado. En un primer momento (pretest) los estudiantes mostraban patrones caracterizados por la "ejecución" en la resolución de los problemas, exploraban las estrategias de resolución y elegían una. Sin embargo, tras la intervención (postest), los estudiantes se decantaron por patrones caracterizados por la "planificación", es decir, identificaban ante qué tipo de problema estaban, anotaban los datos dados en el enunciado, elegían una estrategia para resolverlo y finalmente la llevaban a cabo. En definitiva, la RDI puede favorecer el uso de un proceso de resolución más adecuado, basado en la planificación. Este aspecto, resulta especialmente relevante dado que, tal y como observaron Cueli, García y González-Castro (2013), la planificación es la variable que más diferencia entre grupos de estudiantes con rendimiento bajo y alto. También, los resultados son reseñables dado que tal y como han planteado autores como Gil y Vicent (2009), los primeros años de escolaridad son claves para estimular el desarrollo matemático, ya que es el momento en el que las competencias informales, pueden transformarse en conocimientos y destrezas formales que facilitarán la adquisición de las subsiguientes competencias matemáticas. De ahí, la importancia de que la herramienta vaya dirigida a estudiantes que inician la escolaridad.

Con respecto al segundo de los objetivos específicos, los resultados en las pruebas relacionadas con aspectos atencionales, han mostrado una evolución positiva con una mejora significativa en el postest. Este aspecto, podría relacionarse con los aspectos autorregulatorios trabajados a través del programa, en el que se facilita que el estudiante se capaz de pensar antes, durante y después de que el aprendizaje tenga lugary, en definitiva planificando, ejecutando y revisando (Zimmerman, 2000, 2008). De esta forma, el aprendizaje de estrategias favorece no sólo la realización de tareas matemáticas y la mejora de la competencia a este nivel, sino también, de las habilidades atencionales, las cuales, son modificables y entrenables. En este sentido, 
mejora tanto la percepción de diferencias como la velocidad de nombramiento. Lo cual, resulta especialmente destacable dado que, tal y como exponen Norton y Wolf (2012), las tareas de velocidad de nombrado, son consideradas unas buenas predictoras de aspectos futuros de aprendizaje resultando ser prerrequisitos básicos que facilitan el aprendizaje posterior de habilidades instrumentales. En resumen, la RDI es una herramienta creada para la mejora de la competencia matemática que apunta hacia una buena evolución no sólo sobre la propia competencia matemática, sino también sobre variables no implícitas, como es el caso de la atención, la cual está claramente afectada en el TDAH.

En cualquier caso, es preciso señalar algunas de las limitaciones del presente estudio que deben ser subsanadas en líneas futuras. La primera de ellas, haría referencia a la ausencia de un grupo control que permita la comparación de los resultados obtenidos. Por otro lado, la segunda limitación se encontraría en la baja potencialidad de la muestra, la cual, convendría ampliar con el fin de obtener conclusiones generalizables. Finalmente, sería conveniente que en futuras investigaciones se tuvieran en cuenta los beneficios en función del tipo de presentación de TDAH, con el propósito de estudiar la eficacia de la herramienta RDI en más profundidad.

\section{Conflicto de intereses}

Los autores declaran no tener ningún conflicto de intereses.

\section{REFERENCIAS}

Álvarez, L., González-Castro, P., Núñez, J. C., y González-Pienda, J. A. (2007). Prácticas de Psicología de la educación: evaluación e intervención psicoeducativa. Madrid: Pirámide.

American Psychiatric Association. (2000). Diagnostic and statistical manual of mental disorders-text revision (4th ed.). Washington, DC: Author.

American Psychiatric Association (2013). Diagnostic and statistical manual of mental disorders (DSM-5). Washington, DC: Author.

Arbuckle, J. L. (2010). SPSS Version 19.0. Computer Program. Chicago: SPSS

Cattell, R. B., y Cattell, A. K. S. (1995). Factor G, Escala 1 y Escala 2. Madrid: TEA Ediciones.

Cohen, J. (1988). Statistical power analysis for the behavioral sciences ( $2^{\text {nd }}$ ed.). New Jersey: Lawrence Erlbaum.
Crespo-Eguílaz, N., Narbona, J., Peralta, F., y Repáraz, R. (2014). Medida de atención sostenida y del control de la impulsividad en niños: nueva modalidad de aplicación del Test de Percepción de Diferencias "Caras". Infancia y Aprendizaje, 29(2), 219-232. doi: 10.1174/021037006776789926

Cueli, M., García, T., y González-Castro, P. (2013). Autorregulación y rendimiento académico en matemáticas. Aula Abierta, 4 1(1), 39-48.

De Marco, M. (2010) Programas informáticos para trastornos de lectoescritura, Dislexia y/o TDAH. En Arnaiz, P.; Hurtado, M. D. y Soto, F. J. (Coords.) 25 Años de Integración Escolar en España: Tecnología e Inclusión en el ámbito educativo, laboral y comunitario. Murcia: Consejería de Educación, Formación y Empleo.

DuPaul, G. J., Anastopoulos, A. D., Power, T. J., Reid, R., Ikeda, M. J., y McGoey, K. E. (1998). Parent ratings of attention-deficit/hyperactivity disorder symptoms: Factor structure and normative data. Journal of Psychopathology and Behavioral Assessment, 20, 83102. doi: 10.1023/A:1023087410712

Engel, A., y Onrubia, J. (2013). Estrategias discursivas para la construcción colaborativa del conocimiento en entornos virtuales de aprendizaje. Cultura y Educación, 25(1), 77-94.

Finney, S. J., y Di Stefano, C. (2006). Non-normal and categorical data in structural equation modelling. In G. R. Hancock y R. O. Mueller (Eds.), Structural equation modelling: A second course (pp. 269-314). Greenwich, CT: Information Age.

Gaitán-Chipatecua, A., y Rey-Anacona, C. A. (2013). Diferencias en funciones ejecutivas en escolares normales, con trastorno por déficit de atención, trastorno del cálculo y condición comórbida. Avances en Psicología Latinoamericana, 37(1), $71-85$.

García, T., Rodríguez, C., González-Castro, P., Álvarez, D., Cueli, M., y González-Pienda, J. A. (2013). Funciones ejecutivas en niños y adolescentes con trastorno por déficit de atención con hiperactividad y dificultades lectoras. International Journal of Psychology and Psychological Therapy, 13(2), 179-194.

Gil, M. D., y Vicent, C. (2009). Análisis comparativo de la eficacia de un programa lúdico-narrativo para la enseñanza de las matemáticas en Educación Infantil. Psicothema, $27(1)$, 70-75.

Gioia, G. A., Isquith, P. K., Guy, S. C., y Kenworthy, L. (2000). Behavior Rating Inventory of Executive Function (BRIEF). Lutz, Florida: Psychological Assessment Resources.

González-Castro, P., Cueli, M., Cabeza, L., ÁlvarezGarcía, D., y Rodríguez, C. (2014). Improving basic math skills through integrated dynamic representation strategies. Psicothema, 26, 378-384.

Ginsburg, H. P., y Baroody, A. J. (2003). Test of Early Mathematics Ability (3rd Ed.). Austin, Texas: Pro-Ed.

Kaufmann, L., y Nuerk, H. C. (2008). Basic number processing deficits in ADHD: a broad examination of elementary and complex number processing skills in 9to 12-year-old children with ADHD-C. Developmental Science, 17(5), 692-699. doi: 10.1111/i.14677687.2008.00718.x 
Krawec, J. (2010). Problem Representation and Mathematical Problem Solving of Students of Varying Math Ability. Journal of Learning Disabilities, 47(2), 103-115. doi: 10.1177/0022219412436976

Kroesbergen, E. H., Van Luit, J. E. H., y Aunio, P. (2012). Mathematical and cognitive predictors of the development of mathematics. British Journal of Educational Psychology, 8, 24-27. doi: 10.1111/i.2044-8279.2012.02065.x

Lazakidou, G., y Retalis, S. (2010). Using computer supported collaborative learning strategies for helping students acquire self-regulated problemsolving skills in mathematics. Computers and Education, 54(1), 3-13. https://doi.org/10.1016/i. compedu.2009.02.020

Lucangeli, D., y Cabrele, S. (2006). Mathematical difficulties and ADHD. Exceptionality, 14, 53-62. doi: http://dx.doi.org/10.1207/s15327035ex1401 5

Mayer, R. E. (2004). Psicología de la educación. Madrid: Pearson Educación.

Mayes, S. D., Calhoun, S. L., y Crowell, E. W. (2000). Learning disabilities and ADHD: Overlapping spectrum disorders. Journal of Learning Disabilities, 33,417-424.doi: 10.1177/002221940003300502

Meliá, A. (2008). Dificultades del aprendizaje de las matemáticas en niños con trastorno por déficit de atención e hiperactividad: Comparación de los perfiles cognitivos y metacognitivos. Tesis doctoral, Universidad de Valencia, España.

Miranda, A., Meliá, A. y Marco Taverner, R. (2009). Habilidades matemáticas y funcionamiento ejecutivo de niños con trastorno por déficit de atención con hiperactividad y dificultades del aprendizaje de las matemáticas. Psicothema, 21, 63-69.

Norton, E. S. y Wolf, M. (2012). Rapid Automatized Naming (RAN) and Reading Fluency: Implications for Understanding and Treatment of Reading Disabilities. Annual Review of Psychology, 63, 427-452. doi: 10.1146/annurev-psych-120710-100431
Riley, M., Greeno, J. y Heller, J. (1983). Development of children's problem-solving ability in arithmetic. The development of mathematical thinking (pp. 153196). New York: Academic Press.

Sattler, J. M. y Hoge, R. D. (2008). Evaluación infantil. Aplicaciones conductuales, sociales y clínicas (Garibay, L. D., Trad.). México: Manual Moderno.

Semrud-Clikeman, M. y Bledsoe, J. (2011). Updates on Attention-Deficit/HyperactivityDisorder and Learning Disorders. Current Psychiatry Reports, 13, 364-373. doi: 10.1007/s1 1920-011-0211-5

Servera, M. y Cardo, E. (2007). ADHD Rating Scale-IV en una muestra escolar española: datos normativos y consistencia interna para maestros, padres y madres. Revista de Neurología, 45, 393-399

Solaz-Portolés, J. J., y Sanjosé-López, V. S. (2008). Conocimientos y procesos cognitivos en la resolución de problemas de ciencias: consecuencias para la enseñanza. Magis, Revista Internacional de Investigación en Educación, 1, 147-162.

Thurstone, L. L. y Yela, M. (2012). CARAS-R. Test de Percepción de Diferencias-Revisado. Madrid: Tea Ediciones.

Wolf, M. y Denckla, M.B. (2005). RAN/RAS: Rapid Automatized Naming and Rapid Alternating Stimulus Tests. Austin, Texas: Pro-Ed.

Zimmerman, B. (2000). Attaining self-regulation. A social cognitive perspective. In M. Boekaerts, P. R. Pintrich y M. Zeidner (Eds.), Handbook of selfregulation (pp. 13-39). San Diego, CA: Academic.

Zimmerman, B. (2008). Investigating self-regulation and motivation: historical background, methodological developments, and future prospects. American Educational Research Journal, 45(1), 166 183. doi: $10.3102 / 0002831207312909$

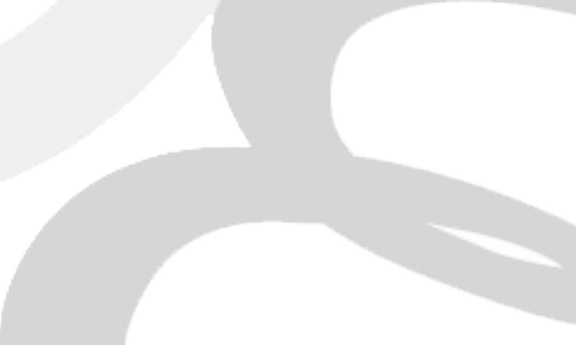

